\title{
DISFUNCIÓN ERÉCTIL DE ORIGEN ESTRUCTURAL PENEANO
}

\author{
Natalio Cruz Navarro
}

Unidad de Urología y Nefrología. Hospital Virgen del Rocío de Sevilla.

Unidad de Andrología. Clínica de Fátima. Sevilla. España.

Resumen.- La delicada armonía con la que los diferentes elementos del pene funcionan se puede ver alterada por diferentes patologías que dañan su estructura. Los fallos estructurales del pene condicionan un fracaso de este evento hemodinámico, y una disfunción eréctil que suele ser severa, resistente al tratamiento médico y generalmente irreversible. La pérdida de complianza en el tejido eréctil un drenaje anómalo de éste o las alteraciones severas en la geometría del pene son los mecanismos fisiopatológicos más frecuentes de estas disfunciones. Entre sus posibles causas figuran, entre otras, la enfermedad de La Peyronie, el priapismo, la diabetes, los traumatismos peneanos y la infiltración de los cuerpos cavernosos por algún proceso inflamatorio o neoplásico.

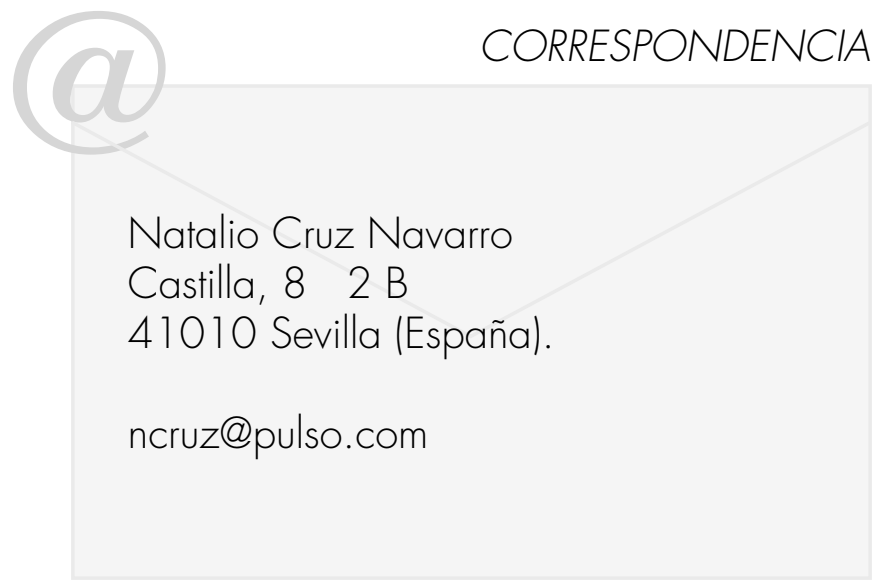

Palabras clave: Mecanismo veno-oclusivo o córporo-venoclusivo. Complianza. Enfermedad de La Peyronie. Priapismo. Uretra. Diabetes. Disfunción eréctil primaria. Prótesis. Disfunción eréctil.

Summary.- The delicate harmony by which the various elements in the penis work may be altered by different pathologies that damage its structure. Structural anomalies in the penis cause a failure of the hemodynamic event and erectile dysfunction, which is usually severe, resistant to medical treatment and generally non reversible. The most frequent physiopathologic mechanisms of these dysfunctions are loss of compliance in the erectile tissue, abnormal drainage, or severe alterations of penile geometry. Among possible etiologies are La Peyronie disease, priapism, diabetes, penile trauma, infiltration of corpora cavernosa by an inflammatory or neoplasic processes, and others.

Keywords: Venous occlusive or corporo-venous occlusive mechanism. Compliance. La Peyronie disease. Priapism. Uretra. Diabetes. Erectile dysfunction.

\section{INTRODUCCIÓN}

Para que una erección se produzca de forma correcta y alcance la rigidez suficiente para penetrar es preciso que haya un llenado de sangre de los cuerpos cavernosos, con una presión intracavernosa suficiente, y que las especiales propiedades estructurales del pene permitan primero su expansión y luego la contención de esa presión hasta su grado máximo.

Los fallos estructurales del pene que pueden conllevar un fracaso de esta armonía hemodinámica, y condicionar una disfunción eréctil serán el objeto de estudio de este apartado. 


\section{MÉTODOS}

Analizaremos en primer lugar los fundamentos anatómicos que permiten la erección y dan rigidez al pene. Recordaremos a continuación algunos conceptos clave de la fisiopatología y sintomatología de los fallos estructurales que condicionarán una disfunción eréctil. Finalmente estudiaremos sus diferentes tipos, deteniéndonos especialmente en los mecanismos etiopatogénicos de los más destacados.

\section{RESULTADOS Y DISCUSIÓN}

\section{Fundamentos anatómicos}

La erección del pene se produce por un aumento de presión dentro del cuerpo cavernoso. Un efecto que al fin y a la postre es fundamentalmente hemodinámico.

Para que ocurra este aumento de presión es necesaria la participación de las arterias helicinas, que aportan sangre al interior de los cuerpos cavernosos; pero también del resto de las estructuras, que ofrecerán resistencia a la salida de esa sangre creando un compartimento de alta presión. El conjunto de estas estructuras que ofrecerán una alta resistencia es lo que denominamos mecanismo veno-oclusivo o córporo-venoclusivo (1).

La primera estructura implicada en el aumento de presión intracavernosa está formada por los propios espacios lacunares del cuerpo cavernoso. Su calibre decreciente progresivamente representa el primer obstáculo a su vaciado y la primera causa del aumento de resistencia, ya que la resistencia vascular es por definición inversamente proporcional al calibre del vaso.

Otras estructuras fundamentales del mecanismo veno-oclusivo son las vénulas subalbugíneas. Cuando el pene se va llenando de sangre estas vénulas cambian su conformación. Se elongan y disminuyen de calibre, aumentando la resistencia a la salida de sangre y con ello la presión intracavernosa. La mayoría incluso se colapsarán completamente en la fase de máxima erección, contribuyendo a alcanzar mayor rigidez.

La tercera estructura importante para alcanzar la rigidez del pene es la propia túnica albugínea, que está perforada por estas vénulas de salida. Juega un papel estructuralmente clave ya que, por un lado debe ser expandirse adecuadamente con la llenada de los cuerpos cavernosos y permitir los cambios de conformación de las vénulas para aumentar la resistencia, y por otro debe poder colapsar estas vénulas para aumentar la resistencia intracavernosa. Además, la túnica albugínea está compuesta por diferentes capas, con fibras de disposición cruzada, lo que contribuye también al mecanismo veno-oclusivo. Esta especial disposición de fibras limita la expansibilidad en un momento determinado y contribuye a la rigidez.

Hay dos cualidades estructurales en la túnica albugínea sana que resultan imprescindibles para alcanzar una completa erección. La primera es la complianza, que permite el llenado del pene, la tumescencia, hasta sus últimas fases. La segunda es su extensibilidad limitada, que condicionará al final de la erección el colapso de las vénulas que la atraviesan y va a permitir alcanzar la rigidez del pene.

Estas dos propiedades se la confiere su composición a base de fibras de colágeno I y III, que son ensanchables pero poco elongables, entrelazadas con fibras de elastina, que pueden elongarse hasta un $150 \%$. Además se compone de varias capas longitudinales y ciculares, con grosores variables a lo largo del pene.

El músculo trabecular es también importante en la regulación del mecanismo veno-oclusivo, ya que para que el pene cambie de volumen y transmita el aumento de presión a la albugínea es necesario que este músculo se relaje. En estado de constricción no se permite el llenado lacunar y no se transmitirá la presión de forma efectiva a la albugínea y no se activará completamente el mecanismo veno-oclusivo (2).

Finalmente, la contracción (voluntaria o involuntaria) durante el coito de los músculos isquio y bulbocavernosos, insertados en la túnica albugínea, contribuirá también al aumento de rigidez. Por un lado porque al traccionar de sus fibras oblicuas ayuda a colapsar las vénulas emisarias que la atraviesan. Por otro porque esta contracción condiciona también una disminución del volumen de los cuerpos cavernosos del pene.

Generalmente, las patologías que afectan a la musculatura trabecular del tejido eréctil suelen causar una disfunción eréctil de tipo funcional, que responde habitualmente bien a fármacos vasoactivos. La relajación farmacológica del músculo permite activarse el resto del mecanismo y posibilita la erección. Por el contrario, las patologías que afectan al resto de los componentes del mecanismo veno-oclusivo condicionan una disfunción eréctil de tipo estructural, que no suele responder bien a fármacos, porque no depende de la relajación del músculo trabecular cavernoso. 


\section{Fisiopatología y sintomatología}

Las alteraciones estructurales que darán lugar a disfunción eréctil pueden ser debidas a una de las dos situaciones siguientes: pérdida de complianza (de la albugínea o del tejido eréctil), o drenaje anómalo de los cuerpos cavernosos (3).

La forma condicionada por la pérdida de complianza va generalmente asociada a procesos de fibrosis, bien como consecuencia de la edad, los factores de riesgo vascular y el reemplazo de tejido eréctil por colágeno y tejido fibrótico, bien de forma secundaria a otros procesos o enfermedades generadoras de fibrosis, como la enfermedad de La Peyronie.

Es propia por tanto de pacientes mayores de cincuenta años. Como consecuencia de la dificultad para activar completamente el mecanismo veno-oclusivo, se presentan como una dificultad paulatina para mantener la erección, erecciones cada vez menos rígidas y detumescencia precoz. La fibrosis de cuerpos cavernosos y de albugínea condicionará en ocasiones alteraciones de la morfología del pene más o menos severas.

La forma de drenaje anómalo es, en cambio, propia de jóvenes. Hay una presencia de canales venosos no colapsables, diferentes a los descritos anteriormente, bien por número, bien por tamaño. Aunque suele haber una compensación hemodinámica, el fracaso veno-oclusivo viene condicionado porque estos canales venosos hacen insuficiente el aporte de sangre por las vías arteriales normales. Se presenta como disfunción eréctil primaria.

Un tercer factor que tiene sin duda un papel importante en la erección es la propia geometría del pene. Dado que la rigidez del pene depende de la estructura de los cuerpos cavernosos, y que ésta depende a su vez de su morfología, un cambio en la geometría de los cuerpos cavernosos puede condicionar una pérdida importante de rigidez. De hecho, se sabe que diferencias en el grosor y longitud del pene condicionan diferencias en la rigidez para una misma presión intracavernosa y expansibilidad (4).

La dependencia de la rigidez de la erección de estos tres factores (presión intracavernosa, geometría del pene y propiedades estructurales de sus componentes) explica las discrepancias clínicohemodinámicas. Estudios hemodinámicas normales pueden corresponder a pacientes sin rigidez adecuada, y pacientes con erección suficiente para penetrar pueden tener estudios hemodinámicas alterados.

\section{Etiopatogenia}

Las principales entidades que pueden causar una disfunción eréctil de origen estructural figuran en la tabla I, y pueden ser clasificadas según su forma de presentación en congénitas o adquiridas. Las primeras se manifiestan generalmente como una disfunción eréctil primaria, mientras que en las adquiridas la disfunción aparece en un sujeto con erecciones previas normales.

\section{Enfermedad de La Peyronie}

En la enfermedad de La Peyronie encontramos una elevada prevalencia de disfunción eréctil. De hecho la práctica totalidad de los pacientes la sufren en algún momento de su evolución. Cuando intentamos objetivarla con métodos diagnósticos encontramos que hasta el $84,6 \%$ de los pacientes presentan tumescencia peneana nocturna alterada (5). Hay trastornos en la eco-doppler color o duplex entre el 65 y el $87,5 \%$ de los pacientes según las series consultadas $(6,7)$. Con velocidades diastólicas finales superiores a $10 \mathrm{~cm} / \mathrm{sg}$ e índices de resistencia inferiores a 0,75, lo que supone disfunción veno-oclusiva. $Y$ el $94,7 \%$ tienen cavernosometrías patológicas, con hallazgos sugestivos de disfunción veno-oclusiva $(8,9)$.

En la etiología de esta enfermedad parece combinarse un estrés mecánico durante la erección en forma de microtraumatismos, con una predisposición genética que condiciona en el tejido eréctil y en la albugínea del cuerpo cavernoso en respuesta a esos traumas una infiltración inflamatoria, la producción anormal de un colágeno de reparación anómalo (menos elástico) y una tendencia generalizada a la fibrosis (10).

Las zonas fibróticas, menos extensibles, condicionarán retracciones de los componentes de los cuerpos cavernosos del pene en erección, tanto longitudinales como transversales, lo que dará lugar a incurvaciones y deformidades circunferenciales con estrechamientos en forma de "reloj de arena". Todas estas deformidades geométricas del pene y la alteración del mecanismo veno-oclusivo impedirán alcanzar una correcta rigidez en la erección cuando la enfermedad está instaurada.

\section{Priapismo}

El priapismo veno-oclusivo se caracteriza por una obstrucción completa o parcial del drenaje de los cuerpos cavernosos. Condiciona un estado de isquemia y acidosis intracavernosa que es muy perjudi- 
cial para el tejido eréctil. La ausencia de óxido nítrico y prostaciclina en el cuerpo cavernoso favorecen la agregación plaquetaria, la formación de trombos en las vénulas de salida y la infiltración trabecular por células inflamatorias. El estado isquémico prolongado llevará a la muerte celular y edema e inflamación intensas y posterior fibrosis en el proceso de reparación tisular $(11,12)$.

Estos cambios estructurales con resultado de fibrosis condicionarán una disfunción eréctil que será más severa cuanto más prolongado haya sido el priapismo (13). En la Tabla II figuran los hallazgos tisulares encontrados con microscopìa electrónica en el priapismo en función del tiempo transcurrido.

La particularidad que tiene el abordaje terapéutico de la disfunción eréctil de estos pacientes es que va a requerir casi en la totalidad de los casos el implante de una prótesis de pene, ya que la fibrosis instaurada hace que la respuesta a otros tratamientos sea escasa. En ese sentido, algunos grupos de trabajo como el St. Peter's Hospitals y el Institute of Urology de Londres, recomiendan el implante precoz, inmediato, de la prótesis. Esto parece evitar la progresión de la fibrosis, hace más fácil el implante y garantiza más la preservación de longitud del pene, con menos complicaciones y más satisfacción para el paciente. Publicaron en 2002 los primeros 8 pacientes manejados así, y más recientemente una serie con 50 casos y excelentes resultados $(14,15)$.

\section{Infiltraciones neoplásicas del pene}

Las neoplasias más frecuentes en el pene afectan fundamentalmente a la piel y tienen una incidencia aproximada del 1 al 7,9 por cada 100.000 hombres. En España el carcinoma epidermoide, su forma más frecuente, representa el $0,7 \%$ de los tumores malignos. Otras formas menos frecuentes son el melanoma, que suele localizarse en glande y prepucio, el carcinoma basocelular, con incidencia anecdótica, y la enfermedad de Paget, que puede afectar a cualquier área genital y requiere biopsia para su diagnóstico (16).

Las neoplasias que afectan a los cuerpos cavernosos son mucho más raras. Pueden causar una disfunción eréctil de origen estructural por infiltración local, por un priapismo, o como consecuencia de su propia evolución y tratamiento. Entre ellas encontramos los sarcomas, que afectan al tejido conectivo y son en su mayoría benignas (linfangioma, hemangioma, fibroma, etc.). También los puede haber malignos como el hemangiopericitoma, leiomiosarcoma y su variedad más agresiva, el fibrosarcoma. Suelen afectar al pene en su zona más proximal y raramente dan metástasis. Se caracterizan por nódulos subcutáneos y requieren la exéresis quirúrgica con amputación parcial o total del pene en la mayoría de los casos (17).

La infiltración de los cuerpos cavernosos por leucemias o linfomas no hodgking suele debutar como un episodio de priapismo de difícil manejo, recurrente y rebelde a todo tipo de medidas. Es de muy mal pronóstico desde el punto de vista funcional. Suele terminar irremisiblemente con una disfunción eréctil irreversible a pesar del correcto tratamiento de la patología primaria (18).

Las metástasis son poco frecuentes en el pene. El $50 \%$ son de origen vesical y prostático, el $25 \%$ son rectales y menos frecuentemente proceden del riñón, páncreas, testículo pulmón, etc. La infiltración metastásica de los cuerpos cavernosos suele manifestarse inicialmente como priapismo, aunque también puede dar directamente disfunción eréctil o ser confundida con una enfermedad de La Peyronie. El tratamiento dependerá del origen del tumor primario, aunque por la naturaleza misma del proceso suelen ser pacientes muy evolucionados y con infausto pronóstico, no sólo funcional eréctil, sino vital (19-23).

\section{Otras infiltraciones no neoplásicas}

Una serie de trastornos metabólicos se asocian con infiltración tisular a distancia. En este contexto se han comunicado anecdóticos casos de disfunción eréctil por infiltración de cuerpos cavernosos en la enfermedad de Fabry (24). O por amiloidosis, que puede infiltrar las estructura neurológicas y provocar una disfunción eréctil por polineuropatía con disfunción autonómica $(25,26)$.

La infiltración infecciosa de los cuerpos cavernosos es una patología rara porque la especial disposición de las estructuras del pene, muy protegido del exterior, y su abundante vascularización los preservan mucho de los agentes infecciosos. Son excepcionales los casos espontáneos (27). Eran más frecuentes cuando se usaban más profusamente las inyecciones intracavernosas como tratamiento de la disfunción eréctil $(28,29)$.

La cavernositis infecciosa tiene un mal pronóstico cara a la erección. Tratada de forma precoz se han descrito algunos casos de preservación de la erección. Sin embargo, su evolución suele ser hacia la resolución cicatricial y fibrosis (30). 


\section{Traumatismos}

Traumatismos de uretra: La rotura traumática uretra se asocia a una disfunción eréctil en el 20-60\% de los casos, especialmente cuando es posterior. Generalmente esta disfunción aparece como consecuencia de los daños estructurales ocasionados por la lesión traumática primaria. En menos casos es secundaria a la cirugía correctora (31). El trauma asociado a fractura ósea incrementa considerablemente el riesgo de DE. Así, hay un $42 \%$ de DE tras lesiones de ramas pélvicas, y hasta un $80 \%$ si hay lesión de las ramas del pubis. Especialmente por lesión vascular (32). Las plastias en estenosis de uretra anterior tienen el mismo riesgo de DE que una intervención de fimosis (33).

Un estudio prospectivo sobre 125 pacientes intervenidos de estenosis uretral con diferentes técnicas evidencia que en el $17 \%$ de las uretroplastias se aprecia una caída del IIEF a los 3 meses de la intervención y una discreta recuperación del 16\% de ellos a los 6 meses. Un análisis de regresión logística de todos los factores implicados pone de manifiesto que el riesgo de DE es superior en los casos de estenosis de uretra posterior y cuando la uretroplastia es

\section{TABLA I. CAUSAS DE DISFUNCIÓN ERÉCTIL DE ORIGEN ESTRUCTURAL.}
Adquiridas:
- Enfermedad de La Peyronie
- Priapismo
- Diabetes
- Infiltraciones neoplásicas
- Traumáticas:
o Fístulas arterio-venosas
o Fractura de pene
- Yatrogenia quirúrgica
- Cavernositis

\section{Congénitas:}
- Incurvación congénita de pene
- Drenaje anómalo de los cuerpos cavernosos
- Hipoplasia de cuerpos cavernosos
- Micropene, estados intersexos y de ambigüedad sexual

término-terminal (34). Sin embargo Barbagli sostiene que esta técnica es segura y no ha encontrado casos de disfunción eréctil en una seria de más de 150 urtroplastias témino-terminales (35).

Traumatismos de pene: Los traumatismos de pene son más frecuentes entre los 15 y 40 años. En líneas generales pueden deberse a dos causas fundamentales, la lesión durante una relación sexual con pene en erección y posición forzada, o la sufrida en deportes de riesgo o por agresión violenta con herida por arma de fuego o arma blanca. En los últimos años, los incidentes de violencia doméstica y la agresividad callejera han disparado su incidencia en las urgencias de los hospitales.

La lesión traumática del pene en erección conlleva generalmente una fractura de la albugínea que suele desencadenar una respuesta reparadora fibrótica, cicatricial, con resultado de cierta disfunción eréctil de origen estructural. El 35\% de los traumatismos cerrados de pene y prácticamente el 100\% de los abiertos conllevan una disfunción eréctil, con fibrosis y curvas en el pene, si no son manejados adecuadamente. El tratamiento quirúrgico precoz de estas roturas puede evitar esta evolución y es el más adecuado (36).

Sólo el 10\% de los pacientes intervenidos de forma precoz tras un traumatismo peneano parecen presentar molestias durante la erección o incurvación. Sin embargo, hasta el $80 \%$ de los no intervenidos presentan disfunción eréctil secundaria a los hematomas, infecciones o fibrosis $(37,38)$.

En los traumatismos cerrados, si estamos ante una contusión de pene sin rotura de albugínea el tratamiento ha de ser conservador. Tras el diagnóstico de rotura de la albugínea se debe plantear siempre la sutura de esta con material reabsorbible.

En los traumatismos abiertos siempre se debe plantear el tratamiento quirúrgico urgente para desbridar tejidos necróticos, minimizar el riesgo de infección y cierre primario de las laceraciones. Se debe dar amplia profilaxis antibiótica, colocación de sonda vesical o catéter suprapúbico si es preciso, y plantear injertos de piel en diferido si la avulsión es extensa. En este caso siempre se pondrán con pene en erección y de espesor medio para evitar las retracciones que dificultarían la erección posterior.

\section{Diabetes}

La prevalencia de la DE en la diabetes es tres veces mayor que en la población general, aparece a 
una edad más temprana y se agrava con la evolución de la enfermedad (39).

Las causas de la DE del diabético son múltiples y variadas. Incluyen una severa disfunción endotelial, con afectación del turnover celular, reducción de eNOS y de la respuesta al oxído nítrico. Por otro lado hay una neuropatía periférica, con cambios degenerativos en nervios parasimpáticos responsables de la erección. Todo ello da lugar a una disfunción eréctil de tipo funcional, con una buena repuesta relativa a fármacos orales y a fármacos vasoactivos intracavernosos. Además, en el cuerpo cavernoso del diabético se han demostrado cambios estructurales que determinan que esta disfunción eréctil sea más permanente. De una parte, hay una lesión derivada de la presencia de radicales libres de oxígeno y especies oxidativas $(40,41)$.

Por otro lado, la hiperglucemia provoca un fenómeno de glicosidación no enzimática de las proteínas contráctiles del músculo liso del cuerpo cavernoso (fenómeno también conocido como glucación). Es un proceso no metabólico (no enzimático), en el que se producen enlaces entre la glucosa y los grupos amino libres de la matriz proteica (lisina, arginina), dando lugar a dos tipos de productos: inicialmente los productos precoces o productos de Amadori; finalmente, los productos finales o avanzados (advanced glycation end products $\circ$ AGE). Son enlaces covalentes entre las proteínas contráctiles, que se producen en momentos de hiperglucemia y son irreversibles. Las proteínas modificadas por estos AGE son de vida media larga, se mantienen alteradas mucho tiempo después de normalizarse la glucemia. El proceso conduce a una fibrosis del cuerpo cavernoso (42).
Los AGE son fuente además de especies reactivas de oxígeno (ROS), son neurotóxicos sobre los nervios nitrérgicos del pene, y se comportan como inhibidores de la eNOS y de la acción vasodilatadora del óxido nítrico (43).

Se ha demostrado con microscopía electrónica que hay también en los diabéticos una reducción del contenido en músculo liso, aumento de los depósitos de colágeno, adelgazamiento de la membrana basal y pérdida de células endoteliales. Evidenciadas tanto en humanos (44), como en experimentación animal (45).

Estos cambios estructurales tienden a producir más fibrosis en el cuerpo cavernoso del diabético. Parecen asociarse a una disfunción más permanente, con peor respuesta a fármacos vasoactivos y peor pronóstico. Desde el punto de vista terapéutico y de la práctica clínica, una vez instaurados, el paciente estará más cerca de la necesidad de un implante de pene. Esto explica además que el implante en los diabéticos implique con frecuencia una cierta dificultad derivada de la fibrosis. De hecho para la cirugía de implante de prótesis en los diabéticos recomendamos contar con dilatadores e incluso cavernotomos, en previsión de dificultades de dilatación de cuerpos cavernosos.

\section{Disfunción eréctil primaria}

En los siguientes procesos, una alteración estructural o geométrica durante la gestación o primera infancia va a conllevar la aparición de una disfunción eréctil primaria. Al tercer mes del desarrollo embrionario, a partir del tubérculo genital, se desarrollan los

TABLA II. HALLAZGOS TISULARES ENCONTRADOS EN EL CUERPO CAVERNOSO CON MICROSCOPÍA ELECTRÓNICA EN EL PRIAPISMO EN FUNCIÓN DEL TIEMPO TRANSCURRIDO.

HALLAZGOS MICROSCOPIA ELECTRONICA:

12 Horas: Destrucción del endotelio sinusoidal

24 Horas: $\quad$ Exposición de la Membrana basal

Adherencia de trombocitos

48 Horas: Trombos en los espacios sinusoidales

Células musculares lisas con Necrosis

Transformación fibroblástica: FIBROSIS 
cuerpos cavernosos y el resto del pene. Las anomalías del desarrollo suelen ser secundarias a déficits de testosterona o de sus receptores.

El micropene se asocia con frecuencia a hipospadias 0 epispadias, a retracciones fibrosas en la base del pene, y a chordae por hipoplasia uretral. Se caracterizan por ser penes simétricos de pequeño tamaño. En menos circunstancias relacionados con déficits endocrinos, en el marco generalmente de estados intersexos.

Las disfunciones eréctiles congénitas pueden tener un origen vascular, por hipoplasia de arterias cavernosas o drenaje anómalo venoso; o ser secundarias a la ausencia de un cuerpo cavernoso, su reemplazo por tejido fibroso o su mal desarrollo.

\section{Abordaje terapeútico}

Este tipo de disfunciones eréctiles en general, como hemos venido apuntando a lo largo del tema, tienen muy mal pronóstico. Responden de forma parcial a los tratamientos con fármacos orales tipo inhibidores de fosfodiesterasas. Los fármacos intracavernosos pueden ser útiles, pero suelen estar contraindicados (especialmente en aquellos con origen fisiopatológico en traumatismos o micro-traumatismos). $Y$ van a precisar un tratamiento quirúrgico en la mayoría de las ocasiones, bien para intentar corregir la alteración anatómica (curva, deformidad), bien para implantar una prótesis.

Hay muchos esfuerzos investigadores encaminados a encontrar sustancias capaces de frenar la fibrosis producida en diferentes entidades clínicas. Así, el efecto negativo del estrés oxidativo y la presencia de radicales libres en la diabetes parece minimizarse mediante el tratamiento preventivo con antioxidantes. Se han empleado en experimentación animal el ácido alfa-lipoico, solo o combinado con el ácido gamma-linolénico, la vitamina $\mathrm{E}$ y el selenito sódico $(46,47)$.

Se encuentran aún en una etapa muy incipiente los esfuerzos por encontrar una forma de obtener mediante Medicina regenerativa una alternativa para sustituir el tejido cavernoso fibrosazo. Tanto la Ingeniería Tisular como la terapia génica se muestran como posibles y prometedoras opciones de futuro para el tratamiento de las deformaciones peneanas y la disfunción eréctil según los trabajos de Hannover $(48,49)$.

\section{CONCLUSIÓN}

La delicada armonía con la que los diferentes elementos del pene funcionan se puede ver alterada por diferentes patologías que dañan su estructura. Los fallos estructurales del pene condicionan un fracaso de esta armonía hemodinámica, y una disfunción eréctil que suele ser severa, resistente al tratamiento médico y generalmente irreversible.

\section{BIBLIOGRAFÍA y LECTURAS RECOMENDADAS ( ${ }^{*}$ lectura de interés $y^{* *}$ lectura fundamental)}

**1. Sáenz de Tejada I, Angulo J, Cellek S, GonzálezCadavid N, Heaton J, Pickard R, Simonsen U. Physiology of erectile function. J Sex Med. 2004 Nov;1(3):254-65

**2. Sáenz de Tejada I.Molecular mechanisms for the regulation of penile smooth muscle contractility. Int J Impot Res. 2002 Feb;14 Suppl 1:S6-10.

3. Sáenz de Tejada I, Angulo J, Cellek S, GonzálezCadavid N, Heaton J, Pickard R, Simonsen U. Pathophysiology of erectile dysfunction. J Sex Med. 2005 Jan;2(1):26-39.

*4. Udelson D, Nehra A, Hatzichristoud D, Krane RJ, Saenz de Tejada I, Goldstein I. Penile rigidity determinants: Engineering análisis of penile buckling forces as a function of corporal soft tissue characteristics, corporal geometry and intracavernosal pressure. Int J Impotence Res. 1994.6,1:5662.

5. Iacono F, Barra S, De Rosa G, Boscaino A, Lotti T. Microestructural disorders of tunica albugínea in patients affected by Peyronie's disease. J Urol.1993.150:1806-9.

6. Montorsi F, Guazzoni G, Bergamaschi F, Consonni P, Rigatti P, Pizzini G, Miani A. Vascular abnormalities in Peyronie's disease: the role of color Doppler sonography. J Urol. 1994 Feb;151(2):373-5.

7. Kadio_lu A, Tefekli A, Erol H, Cayan S, Kandirali E. Color Doppler ultrasound assessment of penile vascular system in men with Peyronie's disease. Int J Impot Res. 2000 Oct;12(5):263-7.

8. Gasior BL, Levine FJ, Wowannesian A, Krane RJ, Goldstein I. Plaque-associeted corporal veno-oclusive dysfunction in idiopathic Peyronie's disease: a pharmacolcavernosometric and pharmacocavernosographic study. World J Urol. 1990.8:90-102.

9. Jordan GH, Angermeier KW. Preoperative evaluation of erectile function with dynamic infusion cavernosometry/cavernosography in patients undergoing surgery for Peyronie's disease: correlation with postoperative results. J Urol. 1993 Oct;150(4):1138-42. 
10. Godec CJ, Van Beek AL. Peyronie's disease is curable. Is it also preventable?. Urology. 1983 Mar;21(3):257-9.

11. Costa WS, Felix B, Cavalcanti AG, Medeiros Jr J, Sampaio FJ. Structural analysis of the corpora cavernosa in patients with ischaemic priapism. BJU Int. 2009 Sep 14.

12. Munarriz R, Park K, Huang YH, Saenz de Tejada I, Moreland RB, Goldstein I, Traish AM. Reperfusion of ischemic corporal tissue: physiologic and biochemical changes in an animal model of ischemic priapism. Urology. 2003 Oct;62(4):760-4

13. Burnett AL, Bivalacqua TJ. Priapism: current principles and practice. Urol Clin North Am. 2007 Nov;34(4):631-42.

14. Rees RW, Kalsi J, Minhas S, Peters J, Kell P, Ralph DJ. The management of low-flow priapism with the immediate insertion of a penile prosthesis. BJU Int. 2002 Dec;90(9):893-7.

15. Ralph DJ, Garaffa G, Muneer A, Freeman A, Rees R, Christopher AN, Minhas S. The Immediate Insertion of a Penile Prosthesis for Acute Ischaemic Priapism. Eur Urol. 2008 Oct 1. [Epub ahead of print]

16. Perea J, Rodríguez Luna JM, Fernández A, Escudero Barrilero A. Carcinoma de pene. Nuestra experiencia y revisión de la literatura. Actas Urol Esp. 1989 Jan-Feb;13(1):24-7.

17. Masson-Lecomte A, Rocher L, Ferlicot S, Benoît G, Droupy S. High-flow Priapism Due to a Malignant Glomus Tumor (Glomangiosarcoma) of the Corpus Cavernosum. Ann Saudi Med. 2009 SepOct;29(5):412

18. Tazi I. Priapism as the first manifestation of chronic myeloid leukemia. J Sex Med. 2009 Nov 13. [Epub ahead of print]

19. Haliloglu AH, Haliloglu N, Akpinar EE, Ataoglu O.Erectile Dysfunction: Initial Symptom of a Patient with Lung Cancer. J Sex Med. 2009 Aug 11.

20. Eguíluz Lumbreras P, Palacios Hernández A, Heredero Zorzo O, Cañada de Arriba F, García García J, Gómez Zancajo VR, Urrutia Avisrror M. Priapismo maligno secundario a cancer de vejiga. Arch Esp Urol. 2009 Apr;62(3):239-42.

21. Celma Domènech $\mathrm{A}$, Planas Morin $\mathrm{J}$, de Torres Ramírez I, Bestard Vallejo J, Mir Maresma C, Morote Robles J. Priapismo secundario a infiltración peneana por cancer vesical. Actas Urol Esp. 2009 Mar;33(3):327-9

22. Park JC, Lee WH, Kang MK, Park SY. Priapism secondary to penile metastasis of rectal cancer. World J Gastroenterol. 2009 Sep 7;15(33):420911

23. Jiang H, Zhu XW, Shi SF, Xie LP, Teng XD, Chen ZD, Zheng XY. Malignant priapism secondary to testicular tumor. Chin Med J (Engl). 2009 Aug 5;122(15):1839-40
24. de Pablo Cárdenas A, Jiménez Calvo JM, Grasa Lanau V, Lozano Uruñuela F, Pinós Paul MA, Montesino Semper M, Sebastián Borruel JL, Barberena Iriberri FJ. Priapismo de alto flujo en edad pediátrica. Revisión. Arch Esp Urol. 1999 Oct;52(8):862-9.

25. Obayashi K, Ando Y, Terazaki H, Yamashita S, Nakagawa K, Nakamura M, Yamashita T, Suga M, Ishizaki T, Uchino M, Ando M. Effect of sildenafil citrate (Viagra) on erectile dysfunction in a patient with familial amyloidotic polyneuropathy ATTR Val30Met. J Auton Nerv Syst. 2000 Apr 12;80(1-2):89-92.

26. Wang AK, Fealey RD, Gehrking TL, Low PA. Patterns of neuropathy and autonomic failure in patients with amyloidosis. Mayo Clin Proc. 2008 Nov;83(11):1226-30.

27. Sood R, Wadhwa SN, Jain V. Neonatal priapism associated with spontaneous bilateral pyocavernositis. Ann Acad Med Singapore. 2006 Jun;35(6):425-7.

28. Vives A, Collado A, Ribé N, Segarra J, Ruiz Castañé E, Pomerol JM. Cavernositis following intracavernous injection of vasoactive drugs. Urol Int. 2001;67(1):111-2.

29. Schwarzer JU, Hofmann R. Purulent corporeal cavernositis secondary to papaverine-induced priapism. J Urol. 1991 Sep;146(3):845-6.

30. Shamloul R, Kamel I. Early treatment of cavernositis resulted in erectile function preservation. $\mathrm{J}$ Sex Med. 2006 Mar;3(2):320-2.

31. Hosseini J, Tavakkoli Tabassi K. Surgical repair of posterior urethral defects: review of literature and presentation of experiences. Urol J. 2008 Fall;5(4):215-22.

32. Armenakas NA, McAninch JW, Lue TF, Dixon CM, Hricak H. Posttraumatic impotence: magnetic resonance imaging and duplex ultrasound in diagnosis and management. J Urol. 1993 May;149(5 Pt 2):1272-5.

33. Coursey JW, Morey AF, McAninch JW, Summerton DJ, Secrest C, White P, Miller K, Pieczonka C, Hochberg D, Armenakas N. Erectile function after anterior urethroplasty. J Urol. 2001 Dec;166(6):2273-6.

34. Xie H, Xu YM, Xu XL, Sa YL, Wu DL, Zhang XC.Asian J Androl. 2009 Mar;11(2):209-14. Evaluation of erectile function after urethral reconstruction: a prospective study. Epub 2009 Jan 5.

35. Barbagli G, De Angelis M, Romano G, Lazzeri M. Long-term followup of bulbar end-to-end anastomosis: a retrospective analysis of 153 patients in a single center experience. J Urol. 2007 Dec;178(6):2470-3. Epub 2007 Oct 15.

36. El Atat R, Sfaxi M, Benslama MR, Amine D, Ayed M, Mouelli SB, Chebil M, Zmerli S. Fracture of the penis: management and long-term results 
of surgical treatment. Experience in 300 cases. J Trauma. 2008 Jan;64(1):121-5.

37. Yapanoglu T, Aksoy Y, Adanur S, Kabadayi B, Ozturk G, Ozbey I. Seventeen years' experience of penile fracture: conservative vs. surgical treatment. J Sex Med. 2009 Jul;6(7):2058-63. Epub 2009 Apr 28.

38. Ibrahiem EH, El-Tholoth HS, Mohsen T, Hekal IA, El-Assmy A. Penile Fracture: Long-term Outcome of Immediate Surgical Intervention. Urology. 2009 Nov 4. [Epub ahead of print]

39. Saenz de Tejada I, Goldstein I. Diabetic penile neuropathy. Urol Clin North Am. 1988 Feb;15(1):1722.

40. Angulo J, González-Corrochano R, Cuevas P, Fernández A, Fuente JM, Rolo F, Allona A, Sáenz de Tejada I. Diabetes Exacerbates the Functional Deficiency of NO/cGMP Pathway Associated with Erectile Dysfunction in Human Corpus Cavernosum and Penile Arteries. J Sex Med. 2009 Nov 12. [Epub ahead of print]

41. Sáenz de Tejada I, Angulo J, Cellek S, GonzálezCadavid N, Heaton J, Pickard R, Simonsen U. Pathophysiology of erectile dysfunction. J Sex Med. 2005 Jan;2(1):26-39.

42. Cameron NE, Cotter MA. Erectile dysfunction and diabetes mellitus: mechanistic considerations from studies in experimental models. Curr Diabetes Rev. 2007 Aug;3(3):149-58. Review.

43. Rodríguez-Mañas L, Angulo J, Vallejo S, Peiró C, Sánchez-Ferrer A, Cercas E, López-Dóriga P, Sánchez-Ferrer CF. Early and intermediate Amadori glycosylation adducts, oxidative stress, and endothelial dysfunction in the strep- tozotocin-induced diabetic rats vasculature. Diabetologia. 2003 Apr;46(4):556-66. Epub 2003 Mar 12.

44. Mersdorf A, Goldsmith PC, Diederichs W, Padula CA, Lue TF, Fishman IJ, Tanagho EA. Ultrastructural changes in impotent penile tissue: a comparison of 65 patients. J Urol. 1991 Apr;145(4):74958.

45. Salama N, Kagawa S. Ultra-structural changes in collagen of penile tunica albuginea in aged and diabetic rats. Int J Impot Res. 1999 Apr;11(2):99105.

46. Keegan A, Cotter MA, Cameron NE. Corpus cavernosum dysfunction in diabetic rats: effects of combined alpha-lipoic acid and gamma-linolenic acid treatment. Diabetes Metab Res Rev. 2001 Sep-Oct;17(5):380-6.

47. Keegan A, Cotter MA, Cameron NE. Effects of diabetes and treatment with the antioxidant alphalipoic acid on endothelial and neurogenic responses of corpus cavernosum in rats. Diabetologia. 1999 Mar;42(3):343-50.

*48. Schultheiss D, Jonas U. Medicina regenerativa en Andrología: Ingeniería Tisular y terapia génica como posibles opciones de tratamiento para las deformaciones peneanas y la disfunción eréctil. Actas Urol Esp. 2006;30(8):801-811.

*49. Udelson D, Nehra A, Hatzichristou DG, Azadzoi K, Moreland RB, Krane J, Saenz de Tejada IS, Goldstein I.Engineering analysis of penile hemodynamic and structural-dynamic relationships: Part I--Clinical implications of penile tissue mechanical properties. Int J Impot Res. 1998 Mar;10(1):15-24. 